\title{
A!
}

This is an electronic reprint of the original article.

This reprint may differ from the original in pagination and typographic detail.

Sigg, Stephan; Palipana, Sameera; Savazzi, Stefano; Kianoush, Sanaz

\section{Capturing Human-Machine Interaction Events from Radio Sensors in Industry 4.0 Environments}

Published in:

Business Process Management Workshops - BPM 2019 International Workshops, Revised Selected Papers

DOI:

10.1007/978-3-030-37453-2_35

Published: 01/01/2019

Document Version

Peer reviewed version

Please cite the original version:

Sigg, S., Palipana, S., Savazzi, S., \& Kianoush, S. (2019). Capturing Human-Machine Interaction Events from Radio Sensors in Industry 4.0 Environments. In C. Di Francescomarino, R. Dijkman, \& U. Zdun (Eds.), Business Process Management Workshops - BPM 2019 International Workshops, Revised Selected Papers (pp. 430435). (Lecture Notes in Business Information Processing; Vol. 362 LNBIP). SPRINGER. https://doi.org/10.1007/978-3-030-37453-2_35

This material is protected by copyright and other intellectual property rights, and duplication or sale of all or part of any of the repository collections is not permitted, except that material may be duplicated by you for your research use or educational purposes in electronic or print form. You must obtain permission for any other use. Electronic or print copies may not be offered, whether for sale or otherwise to anyone who is not an authorised user. 


\title{
Capturing human-machine interaction events from radio sensors in Industry 4.0 environments
}

\begin{abstract}
Stephan Sigg, Sameera Palipana, Stefano Savazzi, Sanaz Kianoush
Abstract. In manufacturing environments, human workers interact with increasingly autonomous machinery. To ensure workspace safety and production efficiency during human-robot cooperation, continuous and accurate tracking and perception of workers' activities is required. The RadioSense project intends to move forward the state-of-the-art in advanced sensing and perception for next generation manufacturing workspace. In this paper, we describe our ongoing efforts towards multi-subject recognition cases with multiple persons conducting several simultaneous activities. Perturbations induced by moving bodies/objects on the electromagnetic wavefield can be processed for environmental perception by leveraging next generation $(5 \mathrm{G}$ ) New Radio (NR) technologies, including MIMO systems, high performance edge-cloud computing and novel (or custom designed) deep learning tools.
\end{abstract}

Keywords: $5 \mathrm{G} \cdot$ Industry $4.0 \cdot$ Radio sensing

\section{Introduction}

We report from ongoing studies in the RadioSense ${ }^{1}$ project (figure 1). The RadioSense project explores passive radio sensing, or vision technologies which aim to track, recognize and analyse human-robot interactions continuously without requiring workers to wear any devices, and without the need for privacy-intrusive video, while ensuring workers' safety and privacy in industrial environments. RadioSense technology leverages real-time collection and processing of ambient (or

\footnotetext{
${ }^{1}$ http://ambientintelligence.aalto.fi/radiosense/
}

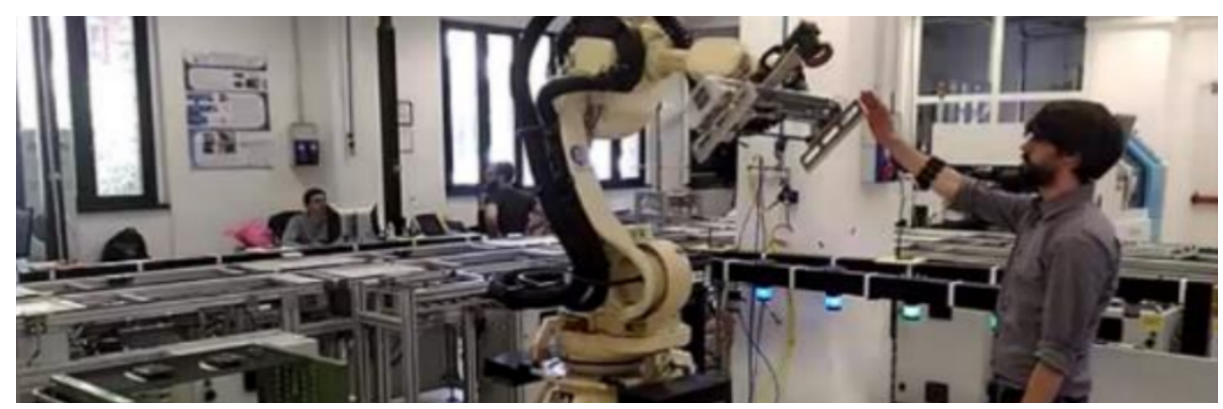

Fig. 1: Industry 4.0 seamless human-robot interaction targeted in RadioSense 


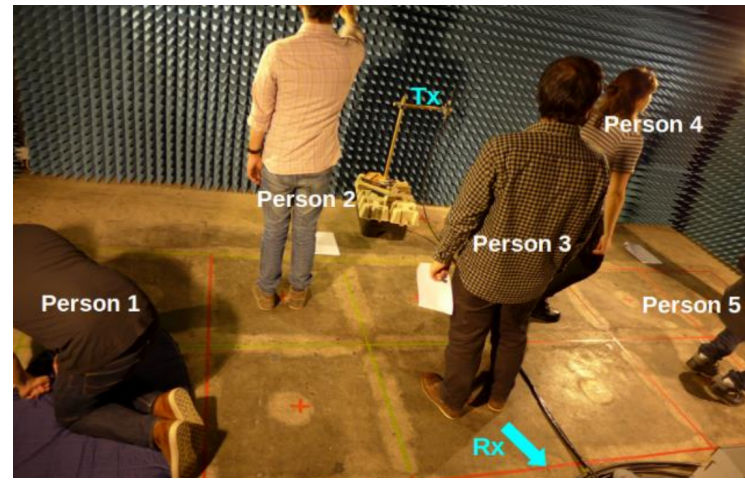

(a) Subjects performing during measurements

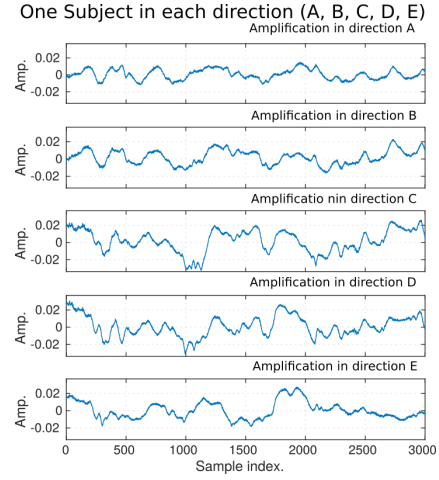

(b) Measured RF signals

Fig. 2: Case study conducted with multiple subjects simultaneously performing distinct activities, gestures and movement.

stray) radio signal streams (e.g., those found in 5G and WiFi connections) and the Channel State Information (CSI) that form a specific type of big data.

In this paper, we describe our ongoing efforts towards multi-subject recognition cases with multiple persons conducting several simultaneous activities. Perturbations induced by moving bodies/objects on the electromagnetic field can be processed for environmental perception. In particular, we will adopt next generation (5G) high-frequency technologies as well as distributed massive MIMO systems.

\section{Multi-subject recognition}

To support human-machine interaction in Industry 4.0 scenarios, we propose to exploit Radio sensing technology [6]. In particular, in the RadioSense project, we investigate installations with multi-antenna receive devices.

A common challenge in traditional radio sensing is to distinguish simultaneous movements from multiple subjects. Essentially, perturbations in the signal strength are typically analyzed at a receiver and interpreted as activities, gestures or other relevant motions. The perturbations are caused by reflection, frequency or phase shift during subject movements. In the case of multiple subjects, reflected signals are superimposed (constructively and destructively combined) and it is not trivial to distinguish individual movements apart. We propose to process the received signal such that each antenna element in the receiver antenna array has a unique phase shift so that signals coming from a given direction is amplified.

Case study We conducted a case study (figure 2a) in an anechoic chamber of size $4 \mathrm{~m} \times 5.2 \mathrm{~m}$ where a signal continuously emitted by a single transmitter was 

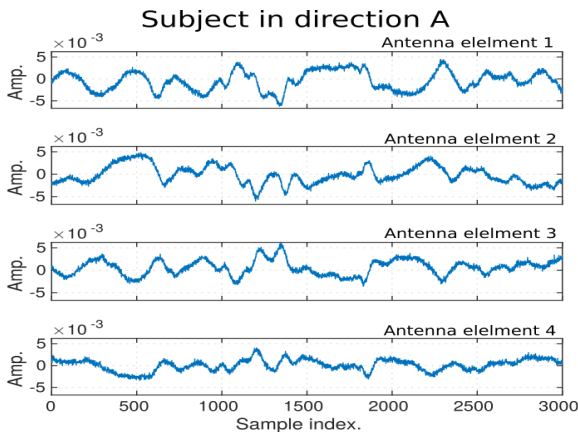

Subject in direction $D$
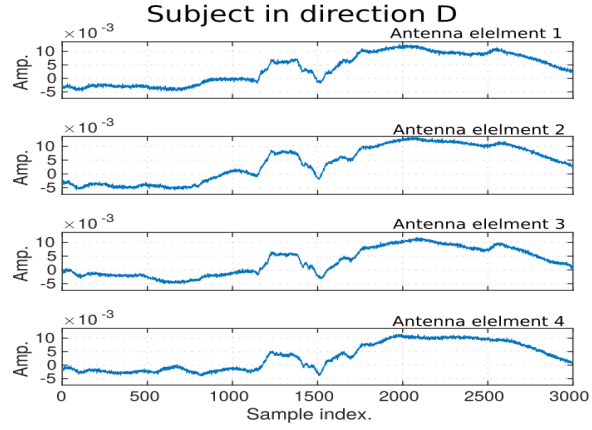
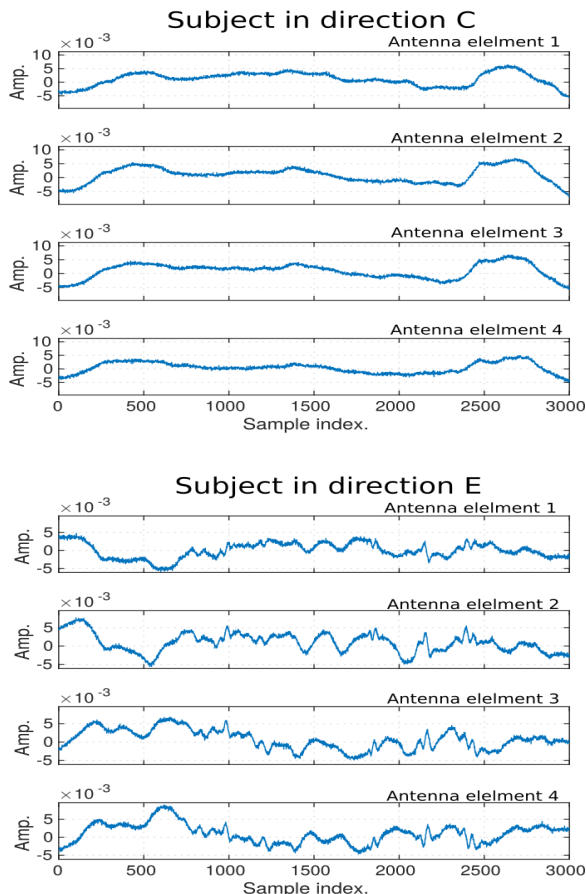

Fig. 3: Raw signal recorded at the four antenna elements while a single subject performs movement in various directions relative to the receive antennas.

captured by a receiver with 4 phase-synchronized linear antenna elements having a spacing of $\frac{\lambda}{2}$ where $\lambda$ is the wavelength at a central frequency of $3.42 \mathrm{GHz}$. In the room, 1 to 5 human subjects performed distinct movements simultaneously while being placed at 5 different locations. We have, in addition, computed 5 sets of phase multipliers for all 4 antennas during the initialization stage to amplify the signals coming from the five directions where the humans are located. In the following, we will refer to these directions as A, B, C, D and E.

Results. For a single subject located in directions A, C, D, or E, figure 3 depicts the signals received at the four receive antennas (for location $\mathrm{B}$, the measurements taken at one of the antennas were omitted as corrupted). We can observe that the signal on all antennas is correlated as all antennas receive the correlated signal perturbations from all directions equally.

While it is possible to distinguish the movement conducted by the subject as demonstrated in the literature (e.g. [5]), subject direction or location is not visible from this time-domain data only ${ }^{2}$. When applying data processing to amplify signal perturbations from respective directions (A, B, C, D, E), we

\footnotetext{
${ }^{2}$ We remark that localization might still be possible from phase information
} 

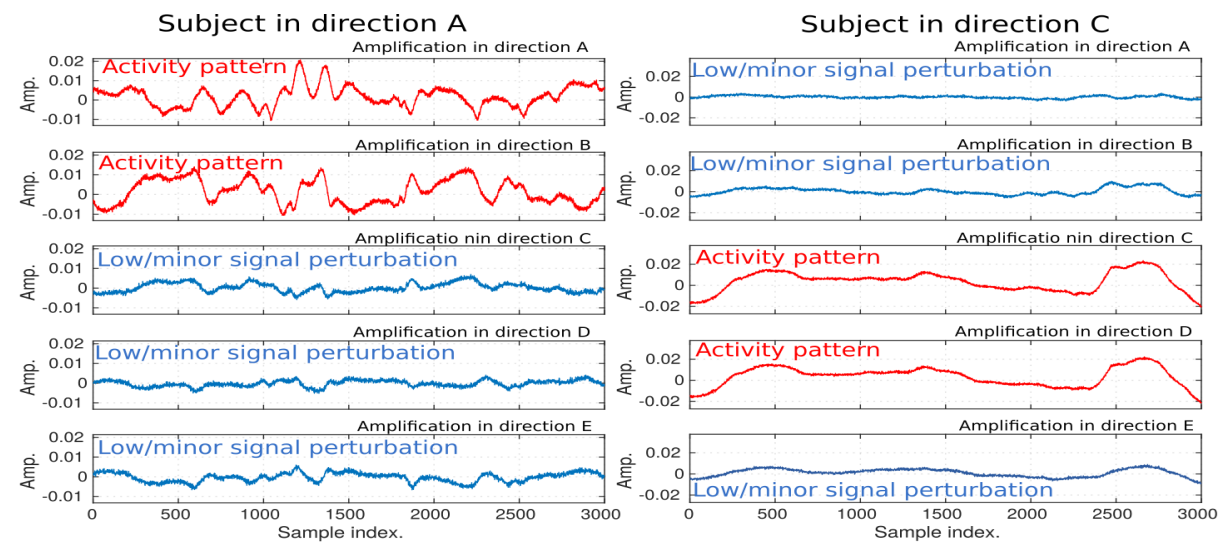

Subject in direction $D$
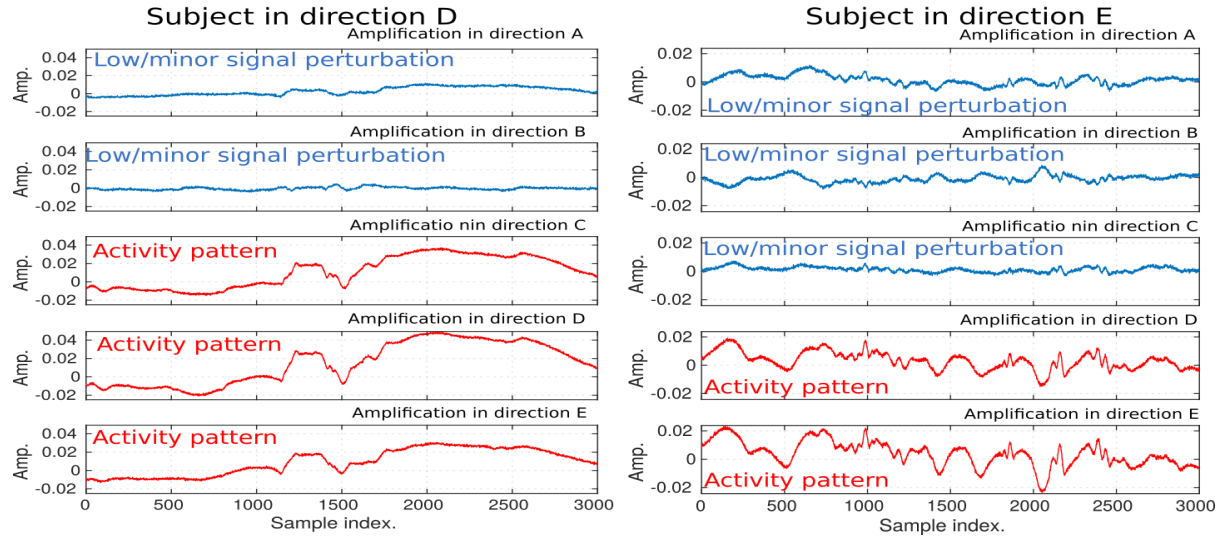

Fig. 4: Directional amplification of signals while a single subject performs movement in various directions relative to the receive antennas.

observe that, for the same data, a rough localization of the subject is possible based on direction of amplified signal (cf. figure 4).

While the accuracy of the directional signal amplification is not sufficient to precisely distinguish between the 5 directions, we observe that only 2-3 adjacent directions are excited with signal perturbations while other locations experience only minor signal perturbation. On top of this, activity recognition might be applied as well as phase-based localization to further improve the accuracy.

Similarly, for multiple (here: two) subjects, figure 5 indicates that distinct patterns are captured from the two subjects from the amplified directions. In contrast, when observing the raw data only (figure 6), no such distinction is possible. Consequently, by amplifying the signals from distinct direction, it shall be possible to recognize distinct workers in Industry 4.0 settings, performing simultaneous activity. We will, in further investigations also study settings with 8,16 or 32 phase-synchronized antennas in order to increase the directional perception accuracy and the count of people that can be recognized simultaneously. 


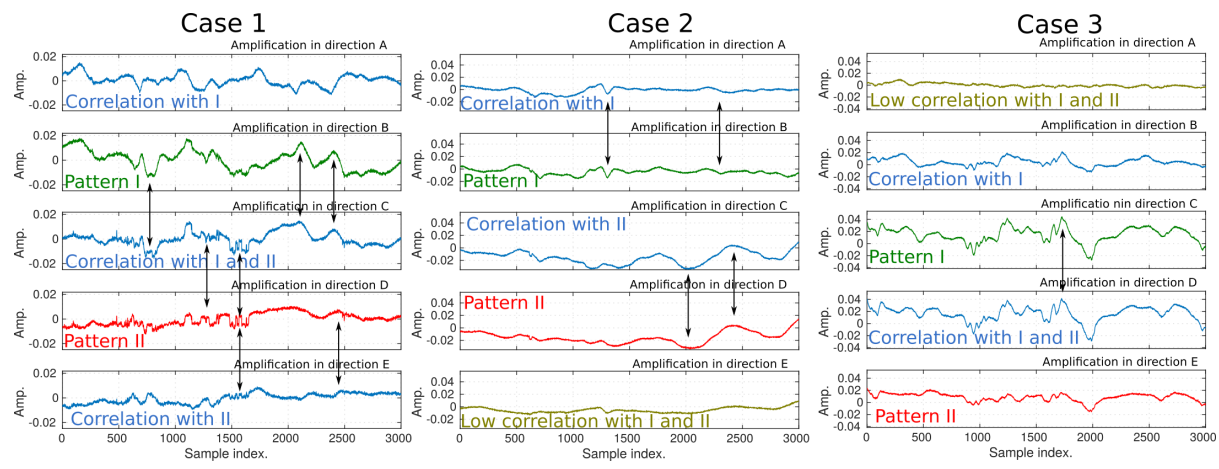

Fig. 5: Two subjects performing movement in different direction (amplification).

In particular, as indicated above already, the perception is limited in the number of individual simultaneous movement that can be recognized by the granularity and accuracy of the environmental perception. In particular, since neighbouring areas are excited too, it was in our setting, for instance, not possible to extract 5 uncorrelated patterns from 5 independently moving subjects at locations A, $\mathrm{B}, \mathrm{C}, \mathrm{D}$, and $\mathrm{E}$. This is depicted in figure $2 \mathrm{~b}$.

\section{Related work}

Research has demonstrated the use of various radio signal measurements for inference of human motion including time delay [1], phase [4], and signal strength [3]; and these have been used for various purposes such as vital sign monitoring [9], activity and gesture recognition [8], localization [7] and fall detection [2].

\section{Discussion and Conclusion}

We have reported from ongoing studies in the RadioSense project regarding the rough relative localization and activity recognition of multiple subjects in Indus-
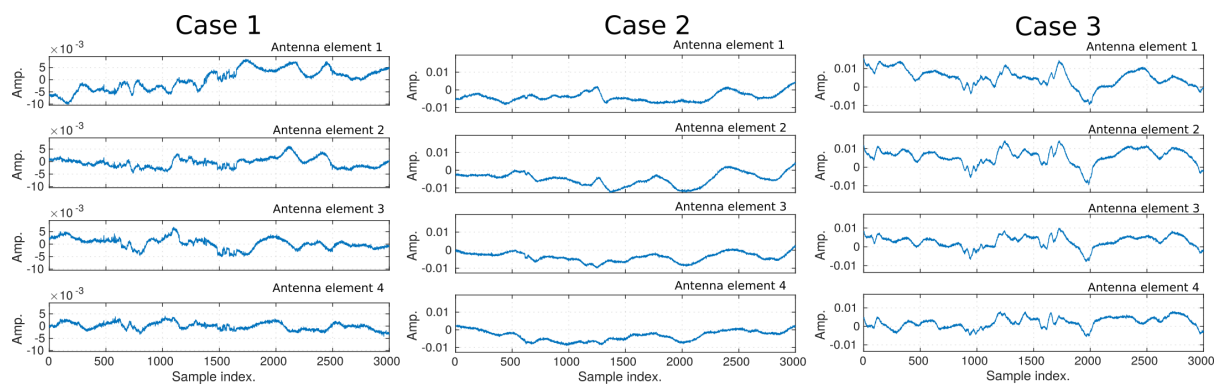

Fig. 6: Two subjects performing movement in different direction (raw). 

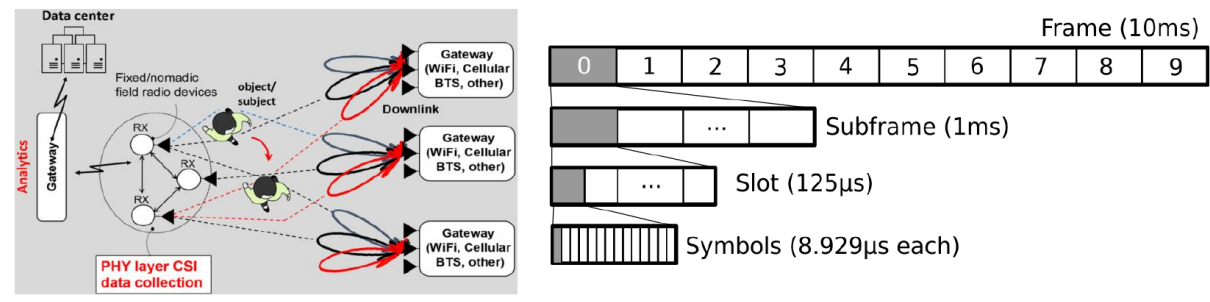

Fig. 7: Integration of Rf-sensing as a service into existing Communication systems

try 4.0 scenarios. For seamless integration into existing environments, we propose the integration of RF-sensing as a service into beyond $5 \mathrm{G}$ cellular systems. A rough calculation (figure 7 ) reveals that the reservation of a single symbol within each subframe of the communication system would translate to a $1 \mathrm{kHz}$ sampling frequency. This is easily sufficient for common activity recognition tasks. At the same time, such allocation would deprive only $1 \%$ of the overall capacity of such communication system from communication to sensing.

Overall, we could show that with appropriate pre-processing of signal phases at distinct, synchronized receive antenna elements, environmental perception in indoor spaces can be improved with respect to relative direction of movement and perception of simultaneously conducted movement.

\section{References}

1. Adib, F., et al.: Smart homes that monitor breathing and heart rate. In: Proc. of CHI (2015)

2. Palipana, S., et al.: FallDeFi: ubiquitous fall detection using commodity Wi-Fi devices. Proc. ACM Interact. Mob. Wearable Ubiquitous Technol. 1(4), 1-25 (2018)

3. Patwari, N., Agrawal, P.: Effects of correlated shadowing: Connectivity, localization, and RF tomography. In: Proc of IPSN (2008)

4. $\mathrm{Pu}, \mathrm{Q}$., et al.: Whole-home gesture recognition using wireless signals. In: Proc. of Mobicom (2013)

5. Savazzi, S., Sigg, S., Vicentini, F., Kianoush, S., Findling, R.: On the use of stray wireless signals for sensing: A look beyond $5 \mathrm{~g}$ for the next generation of industry. Computer 52(7), 25-36 (2019)

6. Savazzi, S., et al.: Device-free radio vision for assisted living: Leveraging wireless channel quality information for human sensing. IEEE Signal Proc. Mag. 33(2), 4558 (2016)

7. Shi, S., et al.: Accurate location tracking from CSI-based passive device-free probabilistic fingerprinting. IEEE Trans. on Veh. Technol. 67(6), 5217-5230 (2018)

8. Sigg, S., et al.: The telepathic phone: Frictionless activity recognition from wifi-rssi. In: Proc of PerCom. pp. 148-155 (2014)

9. Wang, H., et al.: Human respiration detection with commodity wifi devices: Do user location and body orientation matter? In: Proc of Ubicomp (2016) 